\title{
Investigations on the Theory of Riemann Zeta Function II: On the Riemann-Siegel Integral and Hardy's Z-Function
}

\author{
Edigles Guedes ${ }^{1}$, Prof. Dr. Raja Rama Gandhi ${ }^{2}$ and Srinivas Kishan Anapu ${ }^{3}$ \\ ${ }^{1}$ Number Theorist, Pernambuco, Brazil. \\ ${ }^{2}$ Resource person in Math for Oxford University Press, Professor in Math, BITS-Vizag. \\ ${ }^{3}$ CEO, Voice of Bigdata, Austin, TX.
}

ABSTRACT. We create new formulas for Riemann-Siegel Integral and Hardy's Z-function.

\section{INTRODUCTION}

We define Hardy's Z-function to be

$$
Z(t):=e^{i \theta(t)} \zeta\left(\frac{1}{2}+i t\right)
$$

where $\theta(t)$ is given by

$$
\theta(t):=\mathfrak{I}\left(\log \Gamma\left(\frac{1}{4}+\frac{i t}{2}\right)\right)-\frac{t}{2} \log \pi,
$$

see [1, p. 47].

In this paper, we proved a new formula for Hardy's Z-function:

$$
Z(t)=\frac{e^{i \theta(t)}}{2^{\frac{1}{2}+i t}-1}\left[2^{\frac{1}{2}+i t}+\zeta\left(\frac{1}{2}+i t, \frac{3}{2}\right)\right],
$$

and a new formula for Riemann-Siegel integral:

$$
\begin{array}{r}
\int_{C_{N}} \frac{(-x)^{-\frac{1}{2}+i t} e^{-N x}}{e^{x}-1} d x=\frac{e^{2 i \theta(t)}(2 \pi)^{\frac{1}{2}+i t} e^{\frac{t \pi}{2}-\frac{i \pi}{4}}\left(1-i e^{-\frac{i \pi}{4}}\right)}{\left(2^{\frac{1}{2}+i t}-1\right)}\left[2^{\frac{1}{2}+i t}+\zeta\left(\frac{1}{2}+i t, \frac{3}{2}\right)\right] \\
-2(2 \pi)^{\frac{1}{2}+i t} e^{i \theta(t)} e^{\frac{t \pi}{2}-\frac{i \pi}{4}}\left(1-i e^{-\frac{i \pi}{4}}\right) \sum_{n=1}^{N}\left(\frac{\cos (\theta(t)-t \log n)}{n^{\frac{1}{2}}}\right) .
\end{array}
$$

\section{PRELIMINARES}

THEOREM 1. Let $\operatorname{Re}(s)>0$ and $s \neq 1$, then

$$
\zeta(s)=\frac{2^{s}}{2^{s}-1}+\frac{\zeta\left(s, \frac{3}{2}\right)}{2^{s}-1}
$$

where $\zeta(s)$ is the Riemann zeta function and $\zeta(s, a)$ is the Hurwitz zeta function.

Proof. See 2.

COROLLARY 1. For $\operatorname{Re}(s)>0$, then

$$
\left(2^{s}-1\right) \Gamma\left(\frac{1-s}{2}\right) \pi^{s-1 / 2} \zeta(1-s)=\Gamma\left(\frac{s}{2}\right)\left[2^{s}+\zeta\left(s, \frac{3}{2}\right)\right],
$$

and, for $0<\operatorname{Re}(s)<1$, then 


$$
\left(2^{1-s}-1\right) \Gamma\left(\frac{s}{2}\right) \pi^{-s+1 / 2} \zeta(s)=\Gamma\left(\frac{1-s}{2}\right)\left[2^{1-s}+\zeta\left(1-s, \frac{3}{2}\right)\right],
$$

where $\Gamma(s)$ is the gamma function, $\zeta(s)$ is the Riemann zeta function and $\zeta(s, a)$ is the Hurwitz zeta function.

Proof. See [2].

THEOREM 2 (Approximate Functional Equation). Let $x, y \in \mathbb{R}^{+}$with $2 \pi x y=|t|$; then for $s=\sigma+i t$, with $0 \leq \sigma \leq 1$, we have

$$
\zeta(\mathrm{s})=\sum_{\mathrm{n} \leq \mathrm{x}} \frac{1}{\mathrm{n}^{\mathrm{s}}}+\chi(\mathrm{s}) \sum_{\mathrm{n} \leq \mathrm{y}} \frac{1}{\mathrm{n}^{1-\mathrm{s}}}+\mathcal{O}\left(\mathrm{x}^{-\sigma}\right)+\mathcal{O}\left(|\mathrm{t}|^{\frac{1}{2}-\sigma} \mathrm{y}^{\sigma-1}\right),
$$

where $\chi(s)$ is given by

$$
\chi(s)=2^{s} \pi^{s-1} \sin \left(\frac{\pi s}{2}\right) \Gamma(1-s)
$$

Proof. See [1, p. 47].

For the Hardy's Z-function, we have the approximate

$$
Z(t)=2 \sum_{n=1}^{[x]} \frac{\cos (\theta(t)-t \log n)}{n^{\frac{1}{2}}}+\mathcal{O}\left(t^{-\frac{1}{4}}\right)
$$

see $[1$, p. 48].

The Riemann-Siegel Formula. THEOREM 3. For all $t \in \mathbb{R}$,

$$
\mathrm{Z}(\mathrm{t})=2 \sum_{\mathrm{n}=1}^{\mathrm{N}}\left(\frac{\cos (\theta(\mathrm{t})-\mathrm{t} \log \mathrm{n})}{\mathrm{n}^{\frac{1}{2}}}\right)+\frac{\mathrm{e}^{-\mathrm{i} \theta(\mathrm{t})} \mathrm{e}^{-\frac{\mathrm{t} \pi}{2}}}{(2 \pi)^{\frac{1}{2}+\mathrm{it}} \mathrm{e}^{-\frac{\mathrm{i} \pi}{4}}\left(1-\mathrm{i} \mathrm{e}^{-\frac{\mathrm{i} \pi}{4}}\right)} \int_{\mathrm{C}_{\mathrm{N}}} \frac{(-\mathrm{x})^{-\frac{1}{2}+\mathrm{it}} \mathrm{e}^{-\mathrm{Nx}}}{\mathrm{e}^{\mathrm{x}}-1} \mathrm{dx},
$$

where $C_{N}$ is positively oriented closed contour containing all of the points $\pm 2 \pi i N, \pm 2 \pi i(N-$ $1), \ldots, \pm 2 \pi i$, and 0 . See $[1$, p. 48$]$.

\section{LEMMA AND THEOREM}

Hardy's Z-Function. LEMMA 1. For all $t \in \mathbb{R}$, then

$$
Z(t)=\frac{e^{i \theta(t)}}{2^{\frac{1}{2}+i t}-1}\left[2^{\frac{1}{2}+i t}+\zeta\left(\frac{1}{2}+i t, \frac{3}{2}\right)\right] .
$$

Proof. Let $s=\frac{1}{2}+i t$ in $(2.1)$

$$
\zeta\left(\frac{1}{2}+\mathrm{it}\right)=\frac{2^{\frac{1}{2}+\mathrm{it}}}{2^{\frac{1}{2}+\mathrm{it}}-1}+\frac{\zeta\left(\frac{1}{2}+\mathrm{it}, \frac{3}{2}\right)}{2^{\frac{1}{2}+\mathrm{it}}-1} .
$$

Substituting (3.2) in (1.1), we find

$$
Z(t)=\frac{e^{i \theta(t)}}{2^{\frac{1}{2}+i t}-1}\left[2^{\frac{1}{2}+i t}+\zeta\left(\frac{1}{2}+i t, \frac{3}{2}\right)\right] \cdot \square
$$


The Riemann-Siegel Integral. THEOREM 4. For all $t \in \mathbb{R}$, then

$$
\begin{aligned}
& \int_{C_{N}} \frac{(-x)^{-\frac{1}{2}+i t} e^{-N x}}{e^{x}-1} d x=\frac{e^{2 i \theta(t)}(2 \pi)^{\frac{1}{2}+i t} e^{\frac{t \pi}{2}-\frac{i \pi}{4}}\left(1-i e^{-\frac{i \pi}{4}}\right)}{\left(2^{\frac{1}{2}+i t}-1\right)}\left[2^{\frac{1}{2}+i t}+\zeta\left(\frac{1}{2}+i t, \frac{3}{2}\right)\right] \\
&-2(2 \pi)^{\frac{1}{2}+i t} e^{i \theta(t)} e^{\frac{t \pi}{2}-\frac{i \pi}{4}}\left(1-i e^{-\frac{i \pi}{4}}\right) \sum_{n=1}^{N}\left(\frac{\cos (\theta(t)-t \log n)}{n^{\frac{1}{2}}}\right),
\end{aligned}
$$

where $C_{N}$ is positively oriented closed contour containing all of the points $\pm 2 \pi i N, \pm 2 \pi i(N-$ $1), \ldots, \pm 2 \pi i$, and 0 .

Proof. We set (3.3) in the left-hand side of (2.4)

$$
\begin{gathered}
\frac{e^{i \theta(t)}}{2^{\frac{1}{2}+i t}-1}\left[2^{\frac{1}{2}+i t}+\zeta\left(\frac{1}{2}+i t, \frac{3}{2}\right)\right]=2 \sum_{n=1}^{N}\left(\frac{\cos (\theta(t)-t \log n)}{n^{\frac{1}{2}}}\right) \\
+\frac{e^{-i \theta(t)} e^{-\frac{t \pi}{2}}}{(2 \pi)^{\frac{1}{2}+i t} e^{-\frac{i \pi}{4}}\left(1-i e^{-\frac{i \pi}{4}}\right)} \int_{C_{N}} \frac{(-x)^{-\frac{1}{2}+i t} e^{-N x}}{e^{x}-1} d x
\end{gathered}
$$

thereby,

$$
\begin{gathered}
\int_{C_{N}} \frac{(-x)^{-\frac{1}{2}+i t} e^{-N x}}{e^{x}-1} d x=\frac{e^{2 i \theta(t)}(2 \pi)^{\frac{1}{2}+i t} e^{\frac{t \pi}{2}-\frac{i \pi}{4}}\left(1-i e^{-\frac{i \pi}{4}}\right)}{\left(2^{\frac{1}{2}+i t}-1\right)}\left[2^{\frac{1}{2}+i t}+\zeta\left(\frac{1}{2}+i t, \frac{3}{2}\right)\right] \\
-2(2 \pi)^{\frac{1}{2}+i t} e^{i \theta(t)} e^{\frac{t \pi}{2}-\frac{i \pi}{4}}\left(1-i e^{-\frac{i \pi}{4}}\right) \sum_{n=1}^{N}\left(\frac{\cos (\theta(t)-t \log n)}{n^{\frac{1}{2}}}\right) \cdot \square
\end{gathered}
$$

\section{ACKNOWLEDGMENTS}

The first author, Edigles Guedes, thank Prof. Dr. K. Raja Rama Gandhi and your society for their encouragement and support during the development of this paper.

\section{REFERENCES}

[1] Peter Borwein, Stephen Choi, Brendan Rooney, Andrea Weirathmueller, The Riemann Hypothesis -A resource for the afficionado and virtuoso alike, Springer, 2008.

[2] Edigles Guedes and Prof. Dr. Raja Rama Gandhi, Investigations on the Theory of Riemann Zeta Function: New Functional Equation, Integral Representation and Laurent Expansion for Riemann's Zeta Function, May 1, 2013. 